\title{
How diverse are the samples used to study intimate relationships? A systematic review
}

\author{
Hannah C. Williamson, Jerica X. Bornstein, Veronica Cantu, \\ Oyku Ciftci, Krystan A. Farnish, and Megan T. Schouweiler \\ University of Texas at Austin
}

Manuscript accepted for publication in Journal of Social and Personal Relationships

September 30, 2021

\begin{abstract}
Author Note
This article may not exactly replicate the final published version. It is not the copy of record.

Data and analysis code for this project are available at https://osf.io/6pbtk/. An early version of this research was presented at the annual convention of the Society for Personality and Social Psychology (2021).

The authors thank Olivia Ballard, Sarah Carpenter, Elizabeth English, Jisoo Park, and Thao Nguyen for their assistance in coding articles.

Correspondence concerning this article should be addressed to Hannah C. Williamson, 108 E. Dean Keeton St. Stop A2702 Austin, TX 78712. Email: hwilliamson@utexas.edu.
\end{abstract}




\begin{abstract}
The social and behavioral sciences have long suffered from a lack of diversity in the samples used to study a broad array of phenomena. In an attempt to move toward a more contextuallyinformed approach, multiple subfields have undertaken meta-science studies of the diversity and inclusion of underrepresented groups in their body of literature. The current study is a systematic review of the field of relationship science aimed at examining the state of diversity and inclusion in this field. Relationship-focused papers published in five top relationship science journals from 2014-2018 ( $N=559$ articles, containing 771 unique studies $)$ were reviewed. Studies were coded for research methods (e.g., sample source, dyadic data, observational data, experimental design) and sample characteristics (e.g., age, education, income, race/ethnicity, sexual orientation). Results indicate that the modal participant in a study of romantic relationships is 30 years old, White, American, middle-class, college educated, and involved in a different-sex, same-race relationship. Additionally, only 74 studies (10\%) focused on traditionally underrepresented groups (i.e., non-White, low-income, and/or sexual and gender minorities). Findings underscore the need for greater inclusion of underrepresented groups to ensure the validity and credibility of relationship science. We conclude with general recommendations for the field.
\end{abstract}

Key words: couples, intimate relationships, diversity, systematic review 


\section{How diverse are the samples used to study intimate relationships? A systematic review}

The fact that most participants in social and behavioral research are drawn from a small, nonrepresentative subset of people has been known for more than five decades (Schultz, 1969; Smart, 1966). Despite repeated calls throughout the years to increase the diversity and inclusion of underrepresented groups in our samples, the majority of our knowledge about human behavior continues to be drawn from a limited portion of the human population (Bauserman, 1997; Bell \& Hertz, 1976; Gallander Wintre et al., 2001; Graham, 1992; Henrich et al., 2010; Henry, 2008; Sears, 1986). Recent meta-science studies have demonstrated a persistent lack of diversity and inclusion across many fields of psychological and behavioral sciences. For example, $60 \%$ of studies published in six top American Psychological Association journals from 2014-2018 were drawn from the United States, and of these samples $77 \%$ were predominantly European American (Thalmayer et al., 2021). Beyond a lack of racial, ethnic, and cultural diversity, many studies fail to examine anyone other than undergraduate college students: in studies published in the Journal of Personality and Social Psychology, 39\% of the American samples and 54\% of the samples from other countries were composed solely of undergraduate students (Thalmayer et al., 2021).

Although some scholars have argued that the problem of generalizability has been overstated, particularly in more basic areas such as cognition and perception (Haeffel et al., 2009), mounting evidence points to a lack of universality in many social and behavioral phenomena (Henrich et al., 2010). Indeed, recent studies in relationship science have demonstrated group differences in common processes such as demand-withdraw communication and empathic accuracy (Hittner \& Haase, 2021; Ross et al., 2019). Accordingly, examining 
phenomena of interest across diverse participants is necessary to determine whether they are universal or whether they differ across contexts (Apicella et al., 2020).

Recently, multiple meta-science studies have examined subfields of social science to assess the current state of diversity and inclusion, and inform efforts to improve representativeness of study participants within that field (e.g., Nielsen et al., 2017; Pollet \& Saxton, 2019; Qu et al., 2020). The field of relationship science is one area of inquiry that has not yet been scrutinized, yet is ripe for examination of the diversity of participants. On the one hand, romantic relationships are a nearly universal phenomenon, with the vast majority of people around the world engaging in them. On the other hand, there are large differences in the extent to which people engage in specific relationship behaviors such as marriage, cohabitation, divorce, and non-marital childbearing, and the timing of these behaviors, by race/ethnicity, socioeconomic status, and sexual orientation (Allred \& Schweizer, 2020; Manning \& Carlson, 2021; Raley et al., 2015; Sweeney, 2016; Sweeney \& Raley, 2014). More broadly, differences in interpersonal relationships are observed across many cultural and contextual dimensions. For example, seeking interdependence with others vs. maintaining independence from others is one of the defining factors that differentiate between Eastern and Western cultures (Markus \& Kitayama, 1991). Similarly, individuals from different social classes interact differently with others, with lower social class individuals exhibiting more prosocial behaviors, such as trusting and helping others, than their higher social class counterparts (Piff et al., 2010).

The need to understand how intimate relationships function across diverse groups is not just an intellectual exercise; there are also critically important practical implications. For example, in the U.S. Supreme Court decision that legalized same-sex marriage nationwide, the opinion of the court cited an amicus brief filed by the American Psychological Association that 
reviewed the scientific literature on same-sex relationships and argued, among other things, that "gay men and lesbians form stable, committed relationships that are equivalent to heterosexual relationships in essential respects" (Brieffor the APA as Amicus Curiae, Obergefell v. Hodges, 2015). Similarly, the literature on couple relationships was used to justify the establishment of the Healthy Marriage Initiative, a federal policy that has received more than one billion dollars in funding "for the purpose of carrying out healthy marriage promotion activities" primarily aimed at socioeconomically disadvantaged couples (Fein, 2004; Healthy Marriage and Responsible Fatherhood Research and Evaluation, 2020).

Taken together, it is clear that intimate relationships may be influenced by dimensions of human diversity, and that basic relationship science research is already being applied to diverse and underrepresented groups in policy and practice. However, it is not clear the extent to which the field of relationship science has taken heed of these considerations and included diverse and underrepresented samples in basic research on intimate relationships. Although there are no existing comprehensive meta-science studies of relationships, suggestive evidence for a lack of diversity has been mounting for decades. For example, a review published nearly three decades ago which examined the burgeoning longitudinal studies of marriage found that $75 \%$ of these studies used samples comprised primarily of middle-class White participants (Karney \& Bradbury, 1995). Similarly, a meta-analysis of the marriage and relationship education literature published over a decade ago found that only $3 \%$ of studies had predominantly non-White samples, and only $2 \%$ had primarily low-income samples (Hawkins et al., 2008). A paper published in 2004 even provided guidance for how relationship researchers can adjust their sampling practices to garner more diversity within their samples (Karney et al., 2004). 
The current study sought to examine the demographic characteristics of the samples used in relationship science in order to provide an updated and comprehensive assessment of the state of the field. We use the complete corpus of papers published in five of the top relationship science journals from 2014-2018 and assess the extent to which research on intimate relationships has been inclusive of individuals and couples from backgrounds traditionally underrepresented in social and behavioral science. This is an important undertaking for multiple reasons. First, quantifying the areas in which relationship science lacks representation can provide more precise guidance about gaps and future priorities within this field specifically. Next, past meta-science research on the composition of study samples has focused almost exclusively on race/ethnicity and country of origin and overlooked other dimensions of diversity that are centrally relevant to intimate relationships, such as sexual orientation. Additionally, the dyadic nature of intimate relationships means that characteristics of the couple as a unit, such as the gender makeup of the couple or the pairing of individuals into inter-racial relationships, become relevant dimensions to examine within the relationship science literature.

Finally, we move beyond cataloging sample characteristics to examine the type of research being conducted in samples of underrepresented groups. Specifically, in studies that are primarily focused on underrepresented groups, we examine the types of methods and research designs used and the types of research questions asked, and compare this to studies not focused on underrepresented groups. To have a full understanding of intimate relationship processes, we must do more than include underrepresented individuals in our samples. We must also ensure that a rigorous set of research methods (e.g., collection of observational and physiological data) and a full array of research designs (e.g., longitudinal, experimental, qualitative, and daily diary studies) are being used in these samples. And those studies must examine a broad range of 
research questions, including those that test generalizability and those that generate new knowledge.

In sum, the current study systematically reviews papers published in five top relationship science journals from 2014-2018 to address the following aims:

1) Describe the current relationship science literature with respect to features of the samples (i.e., source of the sample, use of dyadic data), research methods (e.g., self-report, observational, physiological), and research designs (i.e., experimental, diary, longitudinal, crosssectional, qualitative).

2) Describe the current relationship science literature with respect to demographics of the samples, including: age, country of origin, education, income, race/ethnicity, sexual orientation and gender diversity, and being part of an inter-racial relationship.

3) Compare the research methods and designs used in studies of underrepresented groups (i.e., low-income individuals, racial/ethnic minorities, and sexual and gender minorities) to those used in studies not focused on underrepresented groups.

4) Examine the types of research questions being addressed in studies focused on underrepresented groups.

Based upon the results of this meta-science study we conclude with recommendations for changes in the field of relationship science to ensure that we are creating a valid and robust understanding of intimate relationships.

\section{Method}

The literature review was begun in early 2019; at that time, we decided to examine the most recent five years' worth of relationship science literature, which included papers published in 2014-2018. We chose five journals that are the top outlets for basic science studies of intimate 
relationships, with the goal of broad coverage of the various disciplines that comprise the interdisciplinary field of relationship science (e.g., social psychology, communication studies, clinical psychology, human development and family sciences): Journal of Family Psychology (JFP), Journal of Marriage and Family (JMF), Journal of Personality and Social Psychology: Interpersonal Relations and Group Processes (JPSP:IRGP), Journal of Social and Personal Relationships (JSPR), and Personal Relationships (PR). The resulting 1,628 papers that were published in these five journals over this five-year period were first assessed for eligibility by reading the abstract, followed by reading the full text if the abstract did not provide definitive information. Papers were eligible for inclusion if they were an empirical study with an intimate relationship-focused dependent variable (e.g., satisfaction, sacrifice, communication, breakup). Papers that had a relationship-focused independent variable but a different dependent variable (e.g., individual physical or mental health, child behavior) were deemed ineligible in order to focus on papers that explicate relationship processes and outcomes, rather than papers that examine sequalae of relationships.

Of 1,628 total papers assessed for eligibility, 1,069 were found to be ineligible and 559 papers had at least one study that was eligible. After removing ineligible studies, we were left with 771 unique studies that met our inclusion criteria for the review (see Figure 1 for the PRISMA flow diagram). Although each study represents a unique dataset within the article in which it was published, it is possible that the same sample was used in multiple articles and thus is counted more than once in our analysis. We attempted to code for the sample source in order to correct for this issue but it was impossible to do so definitively, given the sparse number of details supplied about most of the samples, as detailed in the Results. 
A team of undergraduate research assistants (RAs) coded and extracted data from each of the 771 studies. Each paper was independently coded by two RAs; any assigned code or extracted data that was discrepant between the two coders was resolved by one of the graduate student authors.

\section{Coded Variables}

Sample source. Four different sources from which the sample could be drawn were coded as a Yes or No for whether they were used in the study; each study could be coded as a Yes for multiple sources. The categories were "undergraduate students," “online workers" (e.g., MTurk), "nationally representative sample," and "community sample."

Data type. Each study was coded for whether it used Dyadic data or Individual data.

Research method. Six different categories of research methods were coded as a Yes or No for whether they were used in the study; each study could be coded as a Yes for multiple methods. The categories were "self-report," "observational" (audio or video), "physiological," “interview” (e.g., semi-structured interview, focus group), “administrative data” (e.g., census), and "other" (e.g., informant report, gaze time, Facebook profiles).

Research design. Each study was coded into one of four mutually exclusive research design categories; "experimental," "diary” (which included daily diary, EMA, and time diary), "longitudinal," "cross-sectional," and "qualitative." The experimental category took top precedence, followed by the diary and qualitative categories. Thus, any study that employed an experimental design was coded as experimental, regardless of whether it also included a diary, longitudinal, or cross-sectional design. Next, any study that included a diary or qualitative design was coded as diary or qualitative respectively, regardless of whether it also included a longitudinal design. 
Country. The country from which participants were sampled was recorded. When this was not stated explicitly context clues were used, such as the currency in which participants were paid (e.g., \$70NZD).

Inter-racial couples. The proportion of the sample who were involved in an inter-racial or inter-ethnic couple was recorded.

\section{Constructed Variables}

Age. The average age of participants in each study was recorded. When this value was reported for sample subgroups rather than the full sample (e.g., men and women, patients and partners), a single average age for the sample was calculated. Studies that reported age in some other way (i.e., range, median, frequencies of categories) were coded as yes for providing data on age, but were not included in analyses.

Education. The reported education level of participants was recorded. Because studies typically reported this information categorically, we collapsed the categories to determine the proportion of the sample with the equivalent of a high school diploma or less (i.e., GED, HS diploma, no HS diploma), and the proportion of the sample that had any college education (i.e., associate's degree, some college, bachelor's degree, graduate degree, professional degree). Studies which used exclusively current undergraduate students were coded as having $100 \%$ of participants with some college education. To ensure equivalence of the data, only studies with participants from the United States were coded for this variable. Studies that reported education level in some other way (i.e., mean, mode) were coded as yes for providing data on education, but were not included in analyses.

Geographic region. The country of origin of each study was coded into regions, following categories developed by Arnett (2008) and used in other meta-science research 
(Nielsen et al., 2017; Rad et al., 2018) to facilitate comparison. The categories are; "United States," "English-Speaking Countries" (which is comprised of United Kingdom, Canada, Australia, \& New Zealand), "Europe," “Asia," "Latin America," “Africa," "Middle East,” and "Israel."

Income. The reported income of participants was used to determine the proportion of the sample who were low-income vs. middle-class. Studies of undergraduate students used parental income if it was reported $(n=5)$, or own income $(n=1)$. To ensure equivalence of the data, only studies with participants from the United States were coded for this variable. The threshold for being considered low-income is a household income at or below $200 \%$ of the Federal Poverty Level (FPL), which is based upon year and household size. Year of data collection was used if reported; if not reported, year of publication was used. If income was reported at the individual level, a household size of one was used. If income was reported at the dyad level, a household size of two was used. If the study reported an average number of children for each individual or couple, this number was added to the household size. The year and average household size were used to determine the Federal Poverty Level by consulting the Prior HHS Poverty Guidelines (Office of the Assistant Secretary for Planning and Evaluation, 2021) and this amount was doubled to determine the low-income threshold. For reference, this amount would be $\$ 34,840$ for a household size of two in 2021. The sample mean, sample standard deviation, and samplespecific low-income threshold were entered into an online Normal Distribution Calculator (Lane, n.d.) to determine the proportion of the sample who were below the low-income threshold. Studies were classified as "Primarily Middle-class" if the sample was comprised of <34\% lowincome participants. Studies were classified as "Socioeconomically Diverse" if the sample was comprised of 34-66\% low-income participants. Studies were classified as "Primarily Low- 
Income" if the sample was comprised of $>66 \%$ low-income participants. Studies that reported income in some way other than mean and standard deviation (i.e., range, median, frequencies of categories) were coded as yes for providing data on income, but were not included in analyses of income.

Race/Ethnicity. The proportion of the sample belonging to different racial and ethnic groups was recorded. To ensure equivalence of the data, only studies with participants from the United States were coded for this variable. Based on the way this data was reported across the corpus of studies, the following categories were used and coded for each study as applicable: "Caucasian/White," "Black/African American," "Hispanic/Latino," "American Indian/Alaska Native," "Asian/Pacific Islander/Native Hawaiian," "Middle Eastern/North African," “Biracial/Multiracial/Mixed/Other," "Non-White/Ethnic Minority/Person of Color," "Missing/Declined to report." Because the level of specificity of this information differed across studies, the categories were aggregated into two over-arching categories: "White (NonHispanic)" and "Non-White." Each study that reported information about the race/ethnicity of the participants had a value calculated for the proportion of the sample that was White and the proportion of the sample that was Non-White. These proportions were then used to code studies into three mutually-exclusive categories. Studies were classified as "Primarily White" if the sample was comprised of $<34 \%$ Non-White participants. Studies were classified as "Racially and Ethnically Diverse" if the sample was comprised of 34-66\% Non-White participants. Studies were classified as "Primarily Non-White" if the sample was comprised of $>66 \%$ Non-White participants.

Sexual Orientation and Gender Diversity. The proportion of the sample who were involved in a same-sex relationship, who identified as transgender or gender non-conforming, or 
who were involved in a relationship with an individual who is transgender or gender nonconforming, hereafter referred to as sexual and gender minorities (SGM), was recorded. When this information was not stated explicitly, other information in the manuscript was used to make this determination if possible (e.g., participants were referred to as husbands and wives, indicating that $100 \%$ of the sample were different-sex couples). Studies were classified as an "Over-sample of SGM" if $20 \%$ of more of the sample consisted of individuals from one of these groups. Studies were classified as "Exclusively SGM" if $100 \%$ of the sample consisted of individuals from one of these groups.

\section{Coding of Research Questions Among Studies Focused on Underrepresented Groups}

As a final step, we coded the types of research questions addressed among the subset of studies focused primarily on an underrepresented group (i.e., for race/ethnicity, any study coded as "Primarily Non-White;" for income, any study coded as "Primarily Low-Income;" and for sexual orientation/gender identity any paper coded as an "Over-sample of SGM" or "Exclusively SGM"). Studies meeting this criterion were assigned a yes or no for each of three types of research questions. Categories were not mutually exclusive; each study could have more than one research question and therefore be classified as a yes on more than one type of research question.

A "Basic Science" research question was defined as a question aimed at advancing general knowledge about romantic relationships, without specific consideration of the sample characteristics. A "Generalizability" research question was defined as a question that tests whether a phenomenon that has already been documented in the literature also occurs in this specific group. Finally, a "Population Specific" research question was defined as a question that is targeted toward a specific population of people with the goal of learning more about how this 
specific group operates in their romantic relationships. See Online Supplemental Table 1 for an example of each type of research question. Each study was coded by a minimum of two coders and all discrepancies were resolved by group consensus, with the final decision determined by the first author.

\section{Results}

\section{Sample Source, Geographic Region, and Data Type}

Of the 771 studies, the majority were drawn from the United States $(n=562,73 \%)$, followed by other English-speaking countries (United Kingdom, Canada, Australia, \& New Zealand; $n=90,12 \%)$, and Europe $(n=75,10 \%)$. A small minority of samples came from Asia $(n=25,3 \%)$, Israel $(n=14,2 \%)$, Latin America $(n=3,<1 \%)$ Africa $(n=1,<1 \%)$, and the Middle East $(n=1,<1 \%)$. See Figure 2 . The majority of samples were drawn from the community ( $n=442,57 \%$ ), followed by undergraduate samples, which were the source for $27 \%$ of studies $(n=206)$, and internet samples (i.e., MTurk) which were the source for $11 \%$ of studies $(n=83)$. Nationally representative samples were the least common source of data $(n=46,6 \%)$. Numbers sum to more than 771 because 6 samples used a combination of undergraduate and internet samples. Finally, $42 \%$ of studies used dyadic data $(n=326)$, whereas $58 \%$ of studies used individual data $(n=445)$.

\section{Research Method and Design}

Self-report data was the most commonly used method, with $96 \%$ of studies $(n=743)$ employing it. All other research methods were relatively rare: $11 \%$ of studies used observational data ( $n=86$, including 83 video and 3 audio), $3 \%$ of studies used interview data $(n=17), 2 \%$ of studies used physiological data $(n=17), 2 \%$ of studies used other methods of data collection, such as informant reports or gaze time $(n=14)$, and less than $1 \%$ of studies used administrative 
data $(n=5)$. The most common research design was cross-sectional $(n=348,45 \%)$, followed by longitudinal ( $n=188,24 \%)$, experimental $(n=143,19 \%)$, and diary $(n=71,9 \%$; including 60 daily diary, 6 EMA, and 5 time diary). The least commonly used research design was qualitative $(3 \%, n=21)$.

\section{Demographics of Samples}

Figure 3 presents an overview of the proportion of studies that reported vs. did not report demographic information for age, education, income, being part of an inter-racial couple, race/ethnicity, and sexual orientation and gender identity.

Age. A small proportion of studies $(n=58,8 \%)$ did not provide information about the age of participants. Of the 713 studies that provided information about age, we were able to calculate an average age for 678 studies. Of the remaining 35 studies, age was reported with a statistic other than average $(n=17$ reported a range, $n=9$ reported age categorically, and $n=9$ reported the median). Across the 678 studies, the average age of participants was 30.02 (SD = 11.18), indicating that the majority of participants were in their $20 \mathrm{~s}$ and $30 \mathrm{~s}$. Indeed, $43 \%$ of studies had an average age in the 20 s, followed by $24 \%$ of studies which had an average age in the 30 s. The next most common age group was emerging adults, with $17 \%$ of studies having an average age in the teens. Finally, $10 \%$ of studies had an average in the 40 s, $3 \%$ of studies had an average age in the $50 \mathrm{~s}$, and $3 \%$ of studies had an average age of 60 or higher. These results indicate that adults age 40 and up, who represent nearly half of the U.S. population (U.S Census Bureau, 2020), are being overlooked

Education. A sizable proportion of all 771 studies $(n=277,36 \%)$ did not provide information about the education level of participants. Only the 562 U.S. samples were coded and analyzed on this dimension; the same proportion of U.S. studies did not provide info about 
education level ( $n=201,36 \%$ ). Of the 361 U.S. studies that provided information about education, we were able to use the data from 288 studies in our analyses; the remaining 73 studies reported education in some way other than categorically $(n=37$ reported a mean of years, $n=14$ reported a mean of categories, and $n=3$ reported the mode), used participants who were still engaged in secondary education $(n=8)$, or did not provide sufficient information $(n=$ 11). Of the 288 studies that we were able to include in our analyses, $84 \%$ of participants had some college education or higher and $16 \%$ of participants had a high school diploma or less. For American adults age 25 and older, 59\% have some college education or more (Ryan \& Bauman, 2016), indicating that individuals with higher levels of education are over-represented.

Income. A very large proportion of all 771 studies $(n=583,76 \%)$ did not provide information about the income of participants. Only the 562 U.S. samples were coded and analyzed on this dimension; a slightly smaller proportion of U.S. studies did not provide info about income $(n=402,72 \%)$. Of the 160 U.S. studies that provided information about income, we were able to code 158 studies; the remaining 2 studies did not provide sufficient information to be coded. Across the 158 studies, $64 \%(n=102)$ were a Primarily Middle-class sample, $25 \%$ $(n=39)$ were Socioeconomically Diverse, and $11 \%(n=17)$ were a Primarily Low-income sample. Compared to the U.S. population in which 26\% of Americans are low-income (Semega et al., 2020), these results indicate that Americans living in lower socioeconomic contexts are under-represented.

Race/Ethnicity. A sizable proportion of all 771 studies $(n=260,34 \%)$ did not provide information about the race/ethnicity of participants. Only the 562 U.S. samples were coded and analyzed on this dimension; a smaller proportion of U.S. studies did not provide info about race/ethnicity $(n=130,23 \%)$. Of the 432 U.S. studies that provided information about 
race/ethnicity, we were able to code 427 studies; the remaining 5 studies did not provide sufficient information to be coded. Across the 427 studies, 68\% $(n=290)$ were a Primarily White sample, 21\% ( $n=89)$ were Racially and Ethnically Diverse, and 11\% $(n=48)$ were a Primarily Non-White sample.

Currently, $60 \%$ of the U.S. population is non-Hispanic White (U.S. Census Bureau, 2019), therefore samples that are reflective of this population would fall into the Racially and Ethnically Diverse category. The fact that only $21 \%$ of samples fall into this category, whereas $68 \%$ of sample are Primarily White, indicates that White individuals are being oversampled in comparison to their population prevalence and all other races and ethnicities are being underrepresented.

Inter-racial Couples. The vast majority of studies did not provide information about whether couples were inter-racial/inter-ethnic $(n=708,92 \%)$. Of the 63 studies that reported this information, 38 stated that $0 \%$ of their participants were involved in inter-racial relationships. Thus, 25 studies (3\% of all studies) reported that their sample contained any participants involved in an inter-racial relationship, with an average of $32 \%$ of these samples being interracial couples (range 7\% - 100\%).The number of inter-racial/inter-ethnic couples in the U.S. has been growing in recent decades, and currently $17 \%$ of new marriages are inter-racial or interethnic, indicating that this relationship form is being under-studied in comparison to its prevalence (Livingston \& Brown, 2017).

Sexual Orientation and Gender Diversity. A sizable proportion of all 771 studies $(n=$ $243,32 \%$ ) did not provide information about the sexual orientation or gender identity of the participants. Of the 528 studies that provided information about sexual orientation or gender identity, we were able to code all studies. A total of 21 studies (4\%) were coded as using an 
Oversample or Exclusive Sample of SGM participants. This included nine studies that were an oversample of same-sex couples, nine studies that were an exclusive sample of same-sex couples, and three studies that were an exclusive sample of couples that included a transgender partner. An additional 63 studies (12\%) had any same-sex couples in the sample, with an average of $5 \%$ of these samples consisting of same-sex couples (range $0.21 \%-17.56 \%$ ). In the U.S., $4.5 \%$ of adults identify as lesbian, gay, bisexual, or transgender (Newport, 2018), and $1.5 \%$ of coupled households contain a same-sex couple (Walker \& Taylor, 2021). Based upon the population prevalence, sexual and gender minorities are represented proportionally.

\section{Studies Focused on Underrepresented Samples}

The 17 Primarily Low-income studies, 48 Primarily Non-White studies, and 21 Oversample or Exclusive SGM studies were classified as studies primarily focused on underrepresented groups. This resulted in a total of 74 unique studies because some studies used samples that were underrepresented on more than one dimension (1 study was classified as underrepresented on all 3 dimensions, and 9 studies were underrepresented on race/ethnicity and income). Figure 4 shows the proportion of studies that were focused primarily on one of the three underrepresented groups separately across the three groups. Figure 5 shows the proportion of studies in each of the five journals that were focused on an underrepresented group, collapsed across the three groups.

Sample Sources, Research Methods, and Design. The 74 underrepresented studies were compared against the remaining 697 non-underrepresented studies to examine whether studies focused primarily on underrepresented groups were more likely to draw from particular sample sources or use particular research methods or designs. Chi-square analyses comparing the proportions are presented in Table 1. Compared to studies focused on non-underrepresented 
groups, studies focused on underrepresented groups were significantly more likely to draw the sample from the community, and significantly less likely to draw the sample from undergraduate students and from internet sources (i.e., MTurk). Additionally, studies focused on underrepresented groups were significantly more likely to use dyadic data and equally likely to use observational, interview, and physiological data. Finally, studies focused on underrepresented groups were significantly more likely to use a longitudinal or qualitative research design, significantly less likely to use an experimental design, and equally likely to use a cross-sectional, or diary design.

Research Questions. Of the 17 studies focused primarily on low-income participants, there were 17 distinct research questions. Of these, there were 8 Basic research questions (47\%), 6 Population Specific research questions (35\%), and 0 Generalizability research questions. Additionally, there were 3 research questions stemming from studies that were also underrepresented on another dimension, which were specific to the experience of the other group but not specific to being low-income (2 Specific to non-White and 1 Specific to SGM).

Of the 48 studies focused primarily on non-White participants there were 50 distinct research questions. Of these, there were 29 Basic research questions (58\%), 17 Population Specific research questions (34\%), and 2 Generalizability research questions (4\%). Additionally, there were 2 research questions stemming from studies that were also underrepresented on another dimension, which were specific to the experience of the other group but not specific to being non-White (1 Specific to low-income and 1 Specific to sexual and gender minority).

Of the 21 studies focused primarily on SGM participants there were 26 distinct research questions. Of these, there were 5 Basic research questions (19\%), 18 Population Specific research questions (69\%), and 3 Generalizability research questions (12\%). 


\section{Discussion}

Existing meta-science research has indicated that disciplines across the social sciences suffer from a lack of diversity in their samples (e.g., Rad et al., 2018; Thalmayer et al., 2021). Despite early warnings about the homogenous demographic makeup of samples in relationship science (Karney et al., 2004; Karney \& Bradbury, 1995), the field does not seem to have corrected course. The results of this systematic review indicate that the literature published on intimate relationships in 2014-2018 used samples overwhelmingly drawn from Western countries, with $73 \%$ stemming from the United States and an additional $22 \%$ coming from other Western countries, while only 5\% came from Asia, Africa, Latin America, the Middle East, and Israel combined. This is in line with the broader meta-science literature, which consistently finds that more than $90 \%$ of samples are drawn from Western, industrialized nations (Nielsen et al., 2017; Qu et al., 2020; Thalmayer et al., 2021). Additionally, participants in U.S.-based studies of intimate relationships were overwhelmingly likely to be White, consistent with participant demographics in other disciplines (Nielsen et al., 2017; Qu et al., 2020). These two characteristics (Western vs. non-Western and race/ethnicity) have been the primary focus of the meta-science literature, and the need for more diversity and inclusion across these dimensions is well-established (Cheek, 2017; Dupree \& Kraus, 2021; Hartmann et al., 2013; Henrich et al., 2010; Roberts et al., 2020).

We also move the meta-science literature forward by systematically assessing additional dimensions of human diversity, including socioeconomic status, age, sexual orientation and gender diversity, and inter-racial relationships, topics that, to our knowledge, no other study has examined. Results indicate that people with low-socioeconomic status, older adults, and interracial couples are underrepresented in the relationship science literature compared to their 
prevalence in the population, whereas sexual and gender minorities are represented in the relationship science literature proportional to their population prevalence. However, this group comprises a relatively small proportion of the population, and is itself heterogenous in composition (Watson et al., 2020). Thus, proportional representation in the literature may not be sufficient to build a robust understanding of this (and other minority) groups, a point we return to in the recommendations.

In addition to examining participant characteristics, we also conducted a closer investigation of the subsample of studies that focused primarily on groups who have historically been underrepresented in psychological research: non-White individuals, low-income individuals, and SGM individuals. In terms of research methods and design, studies focused on underrepresented groups were, in general, similarly rigorous to studies not focused on underrepresented groups, with some key differences. Studies focused on underrepresented groups were equally likely to use methods other than self-report (i.e., observational, physiological, interview) and daily diary designs. These studies were more likely to use dyadic data and to draw the sample from the community, and less likely to use undergraduate or online samples. Finally, studies of underrepresented groups were more likely to use a qualitative or longitudinal design, but were less likely to use experimental designs. Taken together, these results suggest that research conducted on underrepresented groups tends to take a more contextual approach by sampling in the community, including both partners, and collecting openended or follow-up data. In contrast, research conducted on non-underrepresented groups appears to prioritize internal validity and causal inference by employing experimental designs, and using undergraduate and online samples (Sue, 1999). 
In terms of the research questions being addressed, studies focused on low-income and non-White participants were skewed more toward answering basic research questions, followed by population specific research questions, with no or few generalizability questions. However, the opposite was true of studies focused on sexual and gender minorities, which skewed toward answering population specific research questions. Taken together, these results suggest that researchers may view the relationships of SGM individuals as more unique and in need of research addressing their specific experiences, compared to the relationships of non-White and low-income individuals, who appear to be seen as a more appropriate population for answering basic questions about intimate relationships. However, we note that these inferences are drawn from a small number of studies $(n=74)$ and should therefore be interpreted with caution. In reality, much more research testing all of these types of research questions in underrepresented groups, using a variety of methods and designs, is needed.

\section{Limitations}

Several factors limit the interpretation of these results. First, our results describing participant demographics are incomplete because of the large amount of missing data. Poor reporting of participant demographics is common (DeJesus et al., 2019; Qu et al., 2020; Rad et al., 2018), and is arguably a bigger problem than reliance on non-diverse samples because the provenance of the results is completely unknown, rather than known to originate in a specific, majority group. Due to this missing data issue, our results are reflective only of the subset of studies that reported participant demographics and not of the entire corpus of studies included in the review. However, we believe that these results are likely to be an accurate representation of sample demographics because it is unlikely that researchers who did not measure and report on 
participant demographics have unintentionally obtained a diverse or underrepresented sample (Karney et al., 2004).

Second, due to the heterogeneity in how participant demographics were reported, we typically had to collapse data to the lowest common denominator and employ coding schemes rather than obtaining precise estimates. This issue is particularly problematic in assessing the race/ethnicity of participants, where we were forced to collapse to a singular "Non-White" category because many studies reported demographics in this manner. This "analytic bifurcation" is problematic because it treats people of color as a single monolithic group and holds up White Americans as the standard to be measured against, and we regret to have to replicate this stance in this work (Williams, 2019).

Finally, it was not feasible to examine every published article across all of relationship science, or to include unpublished work or work published in formats such as books or reports. We chose journals representative of relationship science and its various subdisciplines, but our results may not generalize to other journals, or to books or gray literature.

\section{Recommendations}

Results of this study document multiple issues, including major underreporting of participant demographic data as well as a widespread lack of diversity and inclusion of underrepresented groups across a number of demographic segments. These issues are a threat to the credibility and validity of relationship science because we currently do not know whether the vast majority of our theories and findings generalize beyond a small portion of the human population, and we do not understand the unique relationship processes occurring in nonmajority groups. For relationship science to grow toward a more inclusive and robust field, a number of changes must be made. 
The first and most basic step is to improve reporting of participant demographics in relationship science journals. Reporting standards from various bodies such as the American Psychological Association (followed by JFP, JMF, and JPSP:IRGP) and the International Committee of Medical Journal Editors (followed by JSPR) have long called for reporting of participant demographic characteristics (APA Publications and Communications Board Working Group on Journal Article Reporting Standards, 2008; International Committee of Medical Journal Editors, 2013). However, it is clear that expecting voluntary compliance by authors has not been successful, thus journals must enforce these policies at the editorial level, a practice already used by journals such as Cultural Diversity \& Ethnic Minority Psychology.

The second, and much more difficult, step is to increase the extent to which underrepresented groups are included in relationship science research, and to do so in a manner that is respectful of, and relevant to, these communities. We draw from Hall, Yip, \& Zárate’s (2016) conceptual approach to studying ethnocultural diversity to briefly outline various approaches that researchers may take. The first approach is generalizability research, which simply tests whether a given theory replicates across groups. Generalizability research can draw attention to unstated assumptions of key theories, but typically treat groups as monolithic and provides little information about why results generalize or not. Finding no differences across groups also does not necessarily mean that differences do not exist; post-hoc tests of moderation may be under-powered, or groups may have arrived at the same outcomes via different pathways. The second approach, group differences research, builds on generalizability research by determining potential cultural/contextual mechanisms that are responsible for differences observed across groups. This approach attends more to within-group factors than generalizability research, but still contrasts the underrepresented group against a (typically majority) comparison 
group. The final approach is multicultural research, which involves investigating the mechanisms of cultural/contextual influences on behavior in underrepresented groups. This approach does not use a comparison group, and therefore allows for a fuller understanding of processes within underrepresented groups because research questions are not tied to processes initially observed in majority groups. Multicultural research is particularly important for groups that make up a small proportion of the population, such as sexual and gender minorities, for whom proportional representation within a larger sample would provide under-powered generalizability studies at best.

This brief summary should not serve as a substitute for reading Hall and colleagues (2016) work in full. Indeed, it is important that all researchers take it upon themselves to become culturally-competent and cognizant of the role that their participants' ecological contexts and identities (e.g., age, gender identity, ethnicity, race, sexual orientation, social class), play in their lives (American Psychological Association, 2017). Our results show that even researchers who do not believe themselves to be engaging in "cultural" research are typically studying a particular group - White Americans - who have their own specific cultural values (e.g., Wolfe et al., 2001). In addition to improving cultural competence among researchers, properly conducting multicultural research also requires insights from members of the population of interest in order to avoid deficit narratives and address research questions that are relevant to the population. This can come in the form of diversified research groups who are closely engaged with the population (Nzinga et al., 2018; Roberts et al., 2020), as well as from qualitative research conducted early in the process (Syed, 2018). In fact, though qualitative research made up a very small proportion of the studies in our corpus (3\%), these studies disproportionately focused on underrepresented groups, indicating that a small number of relationship scientists are already using this approach. 
Beyond the practices of individual researchers, larger systemic changes are also needed in our journals and granting agencies. Journal editors and grant reviewers must recognize that samples that come from traditionally underrepresented groups are novel and important contributions to the literature (Rad et al., 2018). Additionally, given the current state of the field, high-quality research on underrepresented groups is likely to include basic observational and descriptive research questions, and these must be valued as important inputs in the research process, alongside experiments that identify causal mechanisms (Syed et al., 2018).

Taking these steps toward diversifying our samples and conducting culturally sensitive research will not just benefit our understanding of underrepresented groups, it will strengthen relationship science as a whole by establishing the foundational knowledge necessary to develop strong, testable theories that will better withstand the test of replication (Scheel et al., 2021). 


\section{References}

Allred, C., \& Schweizer, V. J. (2020). First Divorce Rate in the U.S., 2018 (FP-20-02; Family Profiles). National Center for Family and Marriage Research. https://doi.org/10.25035/ncfmr/fp-20-02

American Psychological Association. (2017). Multicultural Guidelines: An Ecological Approach to Context, Identity, and Intersectionality. http://www.apa.org/about/policy/multiculturalguidelines.pdf

APA Publications and Communications Board Working Group on Journal Article Reporting Standards. (2008). Reporting standards for research in psychology: Why do we need them? What might they be? American Psychologist, 63(9), 839-851. https://doi.org/10.1037/0003-066X.63.9.839

Apicella, C., Norenzayan, A., \& Henrich, J. (2020). Beyond WEIRD: A review of the last decade and a look ahead to the global laboratory of the future. Evolution and Human Behavior, 41(5), 319-329. https://doi.org/10.1016/j.evolhumbehav.2020.07.015

Arnett, J. J. (2008). The neglected 95\%: Why American psychology needs to become less American. American Psychologist, 63(7), 602-614. https://doi.org/10.1037/0003066X.63.7.602

Bauserman, R. (1997). International representation in the psychological literature. International Journal of Psychology, 32(2), 107-112. https://doi.org/10.1080/002075997400908

Bell, R. Q., \& Hertz, T. W. (1976). Toward more comparability and generalizability of developmental research. Child Development, 47(1), 6-13. https://doi.org/10.2307/1128278 
Brief for the APA as Amicus Curiae, Obergefell v. Hodges, (2015). https://www.apa.org/about/offices/ogc/amicus/obergefell-supreme-court.pdf

Cheek, N. N. (2017). Scholarly merit in a global context: The nation gap in psychological science. Perspectives on Psychological Science, 12(6), 1133-1137. https://doi.org/10.1177/1745691617708233

DeJesus, J. M., Callanan, M. A., Solis, G., \& Gelman, S. A. (2019). Generic language in scientific communication. Proceedings of the National Academy of Sciences, 116(37), 18370-18377. https://doi.org/10.1073/pnas.1817706116

Dupree, C. H., \& Kraus, M. W. (2021). Psychological science is not race neutral. Perspectives on Psychological Science, online first publication. https://doi.org/10.1177/1745691620979820

Fein, D. J. (2004). Married and Poor: Basic Characteristics of Economically Disadvantaged Married Couples in the U.S. (Working Paper SHM-01; Supporting Healthy Marriage Evaluation). Abt Associates. https://www.mdrc.org/sites/default/files/workpaper_3.pdf

Gallander Wintre, M., North, C., \& Sugar, L. A. (2001). Psychologists' response to criticisms about research based on undergraduate participants: A developmental perspective. Canadian Psychology, 42(3), 216-225. https://doi.org/10.1037/h0086893

Graham, S. (1992). "Most of the subjects were White and middle class": Trends in published research on African Americans in selected APA journals, 1970-1989. American Psychologist, 47(5), 629-639. https://doi.org/10.1037/0003-066X.47.5.629

Haeffel, G., J., Thiessen, E. D., Campbell, M. W., Kaschak, M. P., \& McNeil, N. M. (2009). Theory, not cultural context, will advance American psychology. American Psychologist, 64(6), 570-571. https://doi.org/10.1037/a0016191 
Hall, G. C. N., Yip, T., \& Zárate, M. A. (2016). On becoming multicultural in a monocultural research world: A conceptual approach to studying ethnocultural diversity. American Psychologist, 71(1), 40-51. https://doi.org/10.1037/a0039734

Hartmann, W. E., Kim, E. S., Kim, J. H. J., Nguyen, T. U., Wendt, D. C., Nagata, D. K., \& Gone, J. P. (2013). In search of cultural diversity, revisited: Recent publication trends in cross-cultural and ethnic minority psychology. Review of General Psychology, 17(3), 243-254. https://doi.org/10.1037/a0032260

Hawkins, A. J., Blanchard, V. L., Baldwin, S. A., \& Fawcett, E. B. (2008). Does marriage and relationship education work? A meta-analytic study. Journal of Consulting and Clinical Psychology, 76(5), 723-734. https://doi.org/10.1037/a0012584

Healthy Marriage and Responsible Fatherhood Research and Evaluation. (2020).

Administration for Children and Families, Office of Family Assistance. https://www.acf.hhs.gov/sites/default/files/documents/opre/Healthy\%20Marriage\%20and \%20Responsible\%20Fatherhood\%20\%28Snapshot\%29-508.pdf

Henrich, J., Heine, S. J., \& Norenzayan, A. (2010). The weirdest people in the world? Behavioral and Brain Sciences, 33(2-3), 61-83. https://doi.org/10.1017/S0140525X0999152X

Henry, P. J. (2008). College sophomores in the laboratory redux: Influences of a narrow data base on social psychology's view of the nature of prejudice. Psychological Inquiry, 19(2), 49-71. https://doi.org/10.1080/10478400802049936

Hittner, E. F., \& Haase, C. M. (2021). Empathy in context: Socioeconomic status as a moderator of the link between empathic accuracy and well-being in married couples. Journal of 
Social and Personal Relationships, 38(5), 1633-1654.

https://doi.org/10.1177/0265407521990750

International Committee of Medical Journal Editors. (2013). Recommendations for the Conduct, Reporting, Editing, and Publication of Scholarly Work in Medical Journals. http://www.icmje.org/recommendations/archives/2013_aug_urm.pdf

Karney, B. R., \& Bradbury, T. N. (1995). The longitudinal course of marital quality and stability: A review of theory, method and research. Psychological Bulletin, 118(1), 3-34. http://dx.doi.org/10.1037/0033-2909.118.1.3

Karney, B. R., Kreitz, M. A., \& Sweeney, K. E. (2004). Obstacles to ethnic diversity in marital research: On the failure of good intentions. Journal of Social and Personal Relationships, 21(4), 509-526. https://doi.org/10.1177/0265407504044845

Lane, D. M. (n.d.). Normal Distribution Calculator. Retrieved April 5, 2021, from https://onlinestatbook.com/2/calculators/normal_dist.html

Livingston, G., \& Brown, A. (2017). Intermarriage in the U.S. 50 Years After Loving v. Virginia. Pew Research Center.

Manning, W., \& Carlson, L. (2021). Trends in Cohabitation Prior to Marriage (FP-21-04; Family Profiles). National Center for Family and Marriage Research. https://doi.org/10.25035/ncfmr/fp-21-04

Markus, H. R., \& Kitayama, S. (1991). Culture and the self: Implications for cognition, emotion, and motivation. Psychological Review, 98(2), 224-253. https://doi.org/10.1037/0033295X.98.2.224

Newport, F. (2018). In U.S., Estimate of LGBT Population Rises to 4.5\%. Gallup. https://news.gallup.com/poll/234863/estimate-lgbt-population-rises.aspx 
Nielsen, M., Haun, D., Kärtner, J., \& Legare, C. H. (2017). The persistent sampling bias in developmental psychology: A call to action. Journal of Experimental Child Psychology, 162, 31-38. https://doi.org/10.1016/j.jecp.2017.04.017

Nzinga, K., Rapp, D. N., Leatherwood, C., Easterday, M., Rogers, L. O., Gallagher, N., \& Medin, D. L. (2018). Should social scientists be distanced from or engaged with the people they study? Proceedings of the National Academy of Sciences, 115(45), 1143511441. https://doi.org/10.1073/pnas.1721167115

Office of the Assistant Secretary for Planning and Evaluation. (2021, March 16). Prior HHS Poverty Guidelines and Federal Register References. https://aspe.hhs.gov/prior-hhspoverty-guidelines-and-federal-register-references

Piff, P. K., Kraus, M. W., Côté, S., Cheng, B. H., \& Keltner, D. (2010). Having less, giving more: The influence of social class on prosocial behavior. Journal of Personality and Social Psychology, 99(5), 771-784. https://doi.org/10.1037/a0020092

Pollet, T. V., \& Saxton, T. K. (2019). How diverse are the samples used in the journals 'Evolution \& Human Behavior' and 'Evolutionary Psychology’? Evolutionary Psychological Science, 5(3), 357-368. https://doi.org/10.1007/s40806-019-00192-2

Qu, Y., Jorgensen, N. A., \& Telzer, E. H. (2020). A call for greater attention to culture in the study of brain and development. Perspectives on Psychological Science, 16(2), 275-293. https://doi.org/10.1177/1745691620931461

Rad, M. S., Martingano, A. J., \& Ginges, J. (2018). Toward a psychology of Homo sapiens: Making psychological science more representative of the human population. Proceedings of the National Academy of Sciences, 115(45), 11401-11405. https://doi.org/10.1073/pnas.1721165115 
Raley, R. K., Sweeney, M. M., \& Wondra, D. (2015). The growing racial and ethnic divide in U.S. marriage patterns. The Future of Children, 25, 89-109. https://doi.org/10.1353/foc.2015.0014

Roberts, S. O., Bareket-Shavit, C., Dollins, F. A., Goldie, P. D., \& Mortenson, E. (2020). Racial inequality in psychological research: Trends of the past and recommendations for the future. Perspectives on Psychological Science, 15(6), 1295-1309. https://doi.org/10.1177/1745691620927709

Ross, J. M., Karney, B. R., Nguyen, T. P., \& Bradbury, T. N. (2019). Communication that is maladaptive for middle-class couples is adaptive for socioeconomically disadvantaged couples. Journal of Personality and Social Psychology, 116(4), 582-597. https://doi.org/10.1037/pspi0000158

Ryan, C. L., \& Bauman, K. (2016). Educational Attainment in the United States: 2015 (No. P20578; Current Population Reports). U.S. Census Bureau.

Scheel, A. M., Tiokhin, L., Isager, P. M., \& Lakens, D. (2021). Why hypothesis testers should spend less time testing hypotheses. Perspectives on Psychological Science, 16(4), 744755. https://doi.org/10.1177/1745691620966795

Schultz, D. P. (1969). The human subject in psychological research. Psychological Bulletin, 72(3), 214-228. https://doi.org/10.1037/ h0027880

Sears, D. O. (1986). College sophomores in the laboratory: Influences of a narrow data base on social psychology's view of human nature. Journal of Personality and Social Psychology, 51(3), 515-530. https://doi.org/10.1037/0022-3514.51.3.515

Semega, J., Kollar, M., Shrider, E. A., \& Creamer, J. F. (2020). Income and Poverty in the United States: 2019 (No. P60-270; Current Population Reports). U.S. Census Bureau. 
Smart, R. G. (1966). Subject selection bias in psychological research. Canadian Psychologist, 7(2), 115-121. https://doi.org/10.1037/h0083096

Sue, S. (1999). Science, Ethnicity, and Bias. American Psychologist, 54(12), 1070-1077. https://doi.org/10.1037/0003-066x.54.12.1070

Sweeney, M. M. (2016). Socioeconomic standing and variability in marriage timing in the twentieth century. The ANNALS of the American Academy of Political and Social Science, 663(1), 270-291. https://doi.org/10.1177/0002716215596975

Sweeney, M. M., \& Raley, R. K. (2014). Race, ethnicity, and the changing context of childbearing in the United States. Annual Review of Sociology, 40, 539-558. https://doi.org/10.1146/annurev-soc-071913-043342

Syed, M., Santos, C., Yoo, H. C., \& Juang, L. P. (2018). Invisibility of racial/ethnic minorities in developmental science: Implications for research and institutional practices. American Psychologist, 73(6), 812-826. https://doi.org/10.1037/amp0000294

Thalmayer, A. G., Toscanelli, C., \& Arnett, J. J. (2021). The neglected 95\% revisited: Is American psychology becoming less American? American Psychologist, 76(1), 116-129. https://doi.org/10.1037/amp0000622

U.S. Census Bureau. (2019). United States Population estimates [QuickFacts]. https://www.census.gov/quickfacts/fact/table/US\#

U.S Census Bureau. (2020). Annual Social and Economic Supplement, 2019 (Current Population Survey).

Walker, L., \& Taylor, D. (2021). Same-Sex Couple Households: 2019 (ACSBR-005; American Community Survey Briefs). U.S. Census Bureau. 
Watson, R. J., Wheldon, C. W., \& Puhl, R. M. (2020). Evidence of diverse identities in a large national sample of sexual and gender minority adolescents. Journal of Research on Adolescence, 30(S2), 431-442. https://doi.org/10.1111/jora.12488

Williams, D. T. (2019). A call to focus on racial domination and oppression: A response to "Racial and ethnic inequality in poverty and affluence, 1959-2015". Population Research and Policy Review, 38(5), 655-663. https://doi.org/10.1007/s11113-019-09538-x

Wolfe, M. M., Yang, P. H., Wong, E. C., \& Atkinson, D. R. (2001). Design and development of the European American values scale for Asian Americans. Cultural Diversity and Ethnic Minority Psychology, 7(3), 274-283. https://doi.org/10.1037/1099-9809.7.3.274 


\section{Table 1}

Comparison of Sample Source, Research Method, and Design of Underrepresented and Nonunderrepresented Samples

\begin{tabular}{lcccc}
\hline & $\begin{array}{c}\text { Non-Underrepresented } \\
\text { Studies }\end{array}$ & $\begin{array}{c}\text { Underrepresented } \\
\text { Studies }\end{array}$ & $\chi^{2}(1)$ & $p$ \\
\cline { 2 - 5 } Community Sample & $55 \%$ & $81 \%$ & 18.88 & $<.001$ \\
Undergraduate Sample & $28 \%$ & $15 \%$ & 5.87 & .015 \\
Internet Sample & $12 \%$ & $3 \%$ & 5.54 & .019 \\
Dyadic Data & $41 \%$ & $54 \%$ & 4.65 & .031 \\
Observational Data & $11 \%$ & $12 \%$ & 0.01 & .906 \\
Physiological Data & $2 \%$ & $1 \%$ & 0.28 & .599 \\
Interview Data & $2 \%$ & $8 \%$ & 13.23 & $<.001$ \\
Cross-Sectional Design & $45 \%$ & $42 \%$ & 0.35 & .555 \\
Longitudinal Design & $23 \%$ & $35 \%$ & 5.96 & .003 \\
Experimental Design & $20 \%$ & $9 \%$ & 5.13 & .023 \\
Diary Design & $10 \%$ & $5 \%$ & .032 \\
\hline
\end{tabular}

Note. $n=697$ Non-Underrepresented Studies, $n=74$ Underrepresented Studies 
Figure 1

PRISMA Flow Diagram
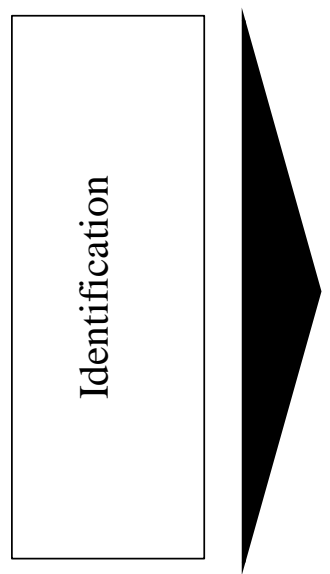

\begin{tabular}{|c|c|c|c|c|c|}
\cline { 2 - 6 } \multicolumn{1}{c|}{} & \multicolumn{5}{c|}{$\begin{array}{c}\text { Comprehensive Literature Review } \\
\text { (January 2014 - December 2018) }\end{array}$} \\
\cline { 2 - 7 } & $P R$ & $J S P R$ & $J M F$ & $J F P$ & $\begin{array}{c}J P S P: \\
I R G P\end{array}$ \\
\hline All Published Articles & $n=229$ & $n=299$ & $n=395$ & $n=522$ & $n=183$ \\
\hline
\end{tabular}

Total Articles to be Screened $(N=1,628)$

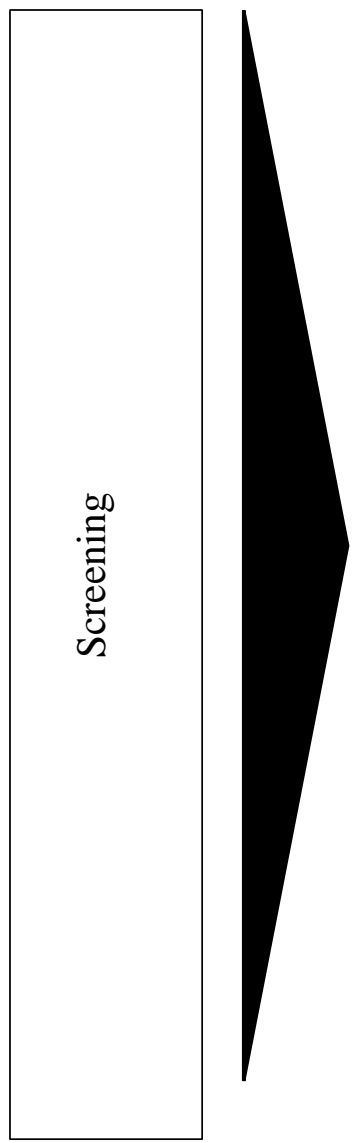

\begin{tabular}{|c|c|c|c|c|}
\hline \multicolumn{5}{|c|}{ Reasons Articles were Excluded After Screening $(N=1,069)$} \\
\hline \multicolumn{5}{|c|}{ Adolescent Outcomes $(n=99)$} \\
\hline \multicolumn{5}{|c|}{ Adult Outcomes $(n=364)$} \\
\hline \multicolumn{5}{|c|}{ Child Outcomes $(n=139)$} \\
\hline \multicolumn{5}{|c|}{ Family Outcomes $(n=95)$} \\
\hline \multicolumn{5}{|c|}{ Gender Roles $(n=27)$} \\
\hline \multicolumn{5}{|c|}{ Intergenerational Relations $(n=72)$} \\
\hline \multicolumn{5}{|c|}{ Literature Review $(n=9)$} \\
\hline \multicolumn{5}{|c|}{ Meta-analysis $(n=5)$} \\
\hline \multicolumn{5}{|c|}{ Methodology $(n=74)$} \\
\hline \multicolumn{5}{|c|}{ Non-Couple Relationships $(n=25)$} \\
\hline \multicolumn{5}{|c|}{ Parenting $(n=149)$} \\
\hline \multicolumn{5}{|c|}{ Singlehood $(n=6)$} \\
\hline \multicolumn{5}{|c|}{ Theory $(n=5)$} \\
\hline \multicolumn{5}{|c|}{$\begin{array}{l}\text { Articles eligible for coding }(N=559) \\
\text { Containing } N=771 \text { studies }\end{array}$} \\
\hline \multicolumn{5}{|c|}{$N=771$ studies } \\
\hline PR & JSPR & JMF & JFP & JPSP: IRGP \\
\hline$n=193$ & $n=175$ & $n=103$ & $n=162$ & $n=138$ \\
\hline
\end{tabular}


Figure 2

Geographic Representation of Studies

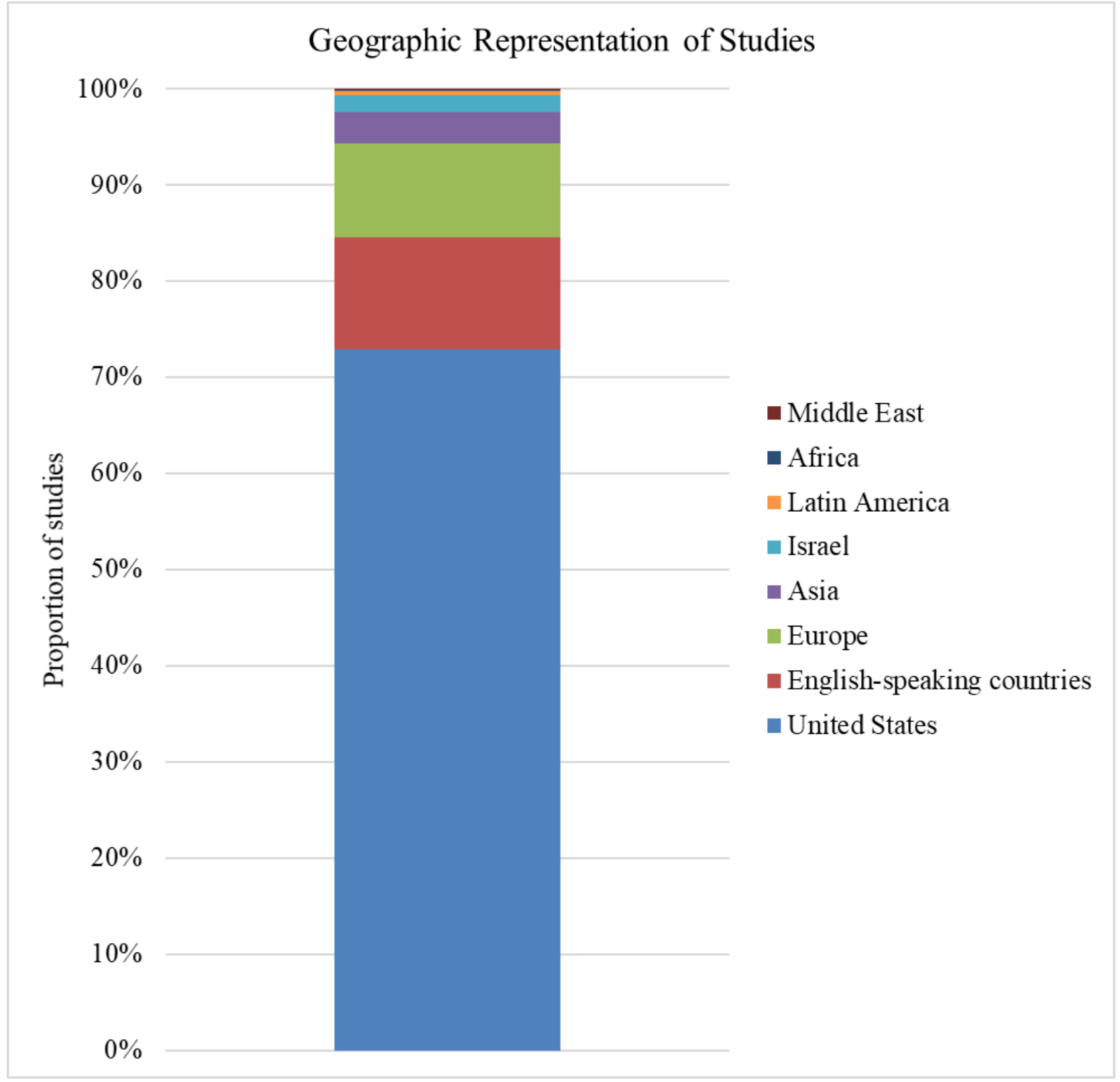


Figure 3

Proportion of Studies that Reported vs. Did Not Report Key Demographics

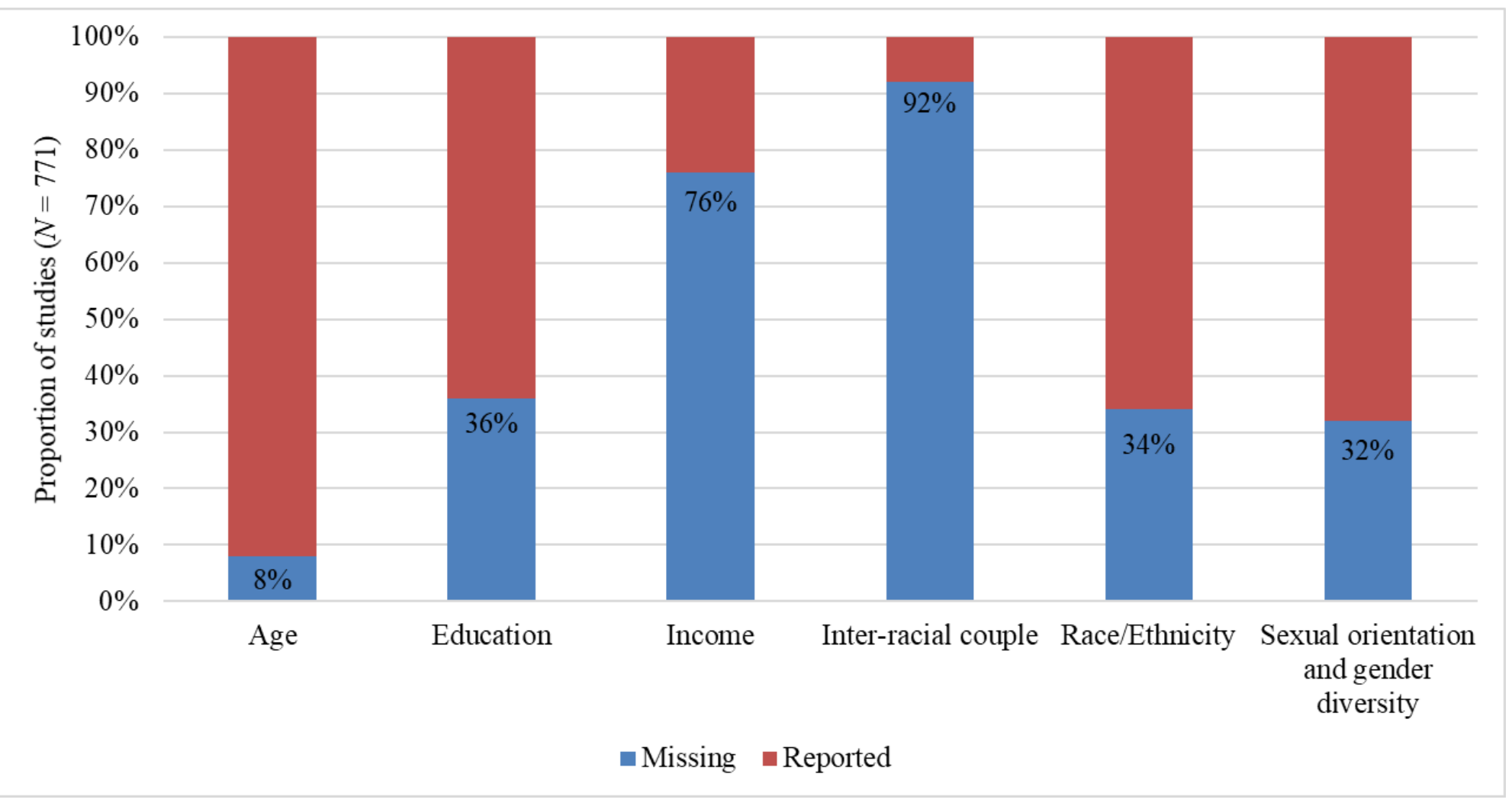




\section{Figure 4}

Proportion of Studies that Focused Primarily on an Underrepresented Group

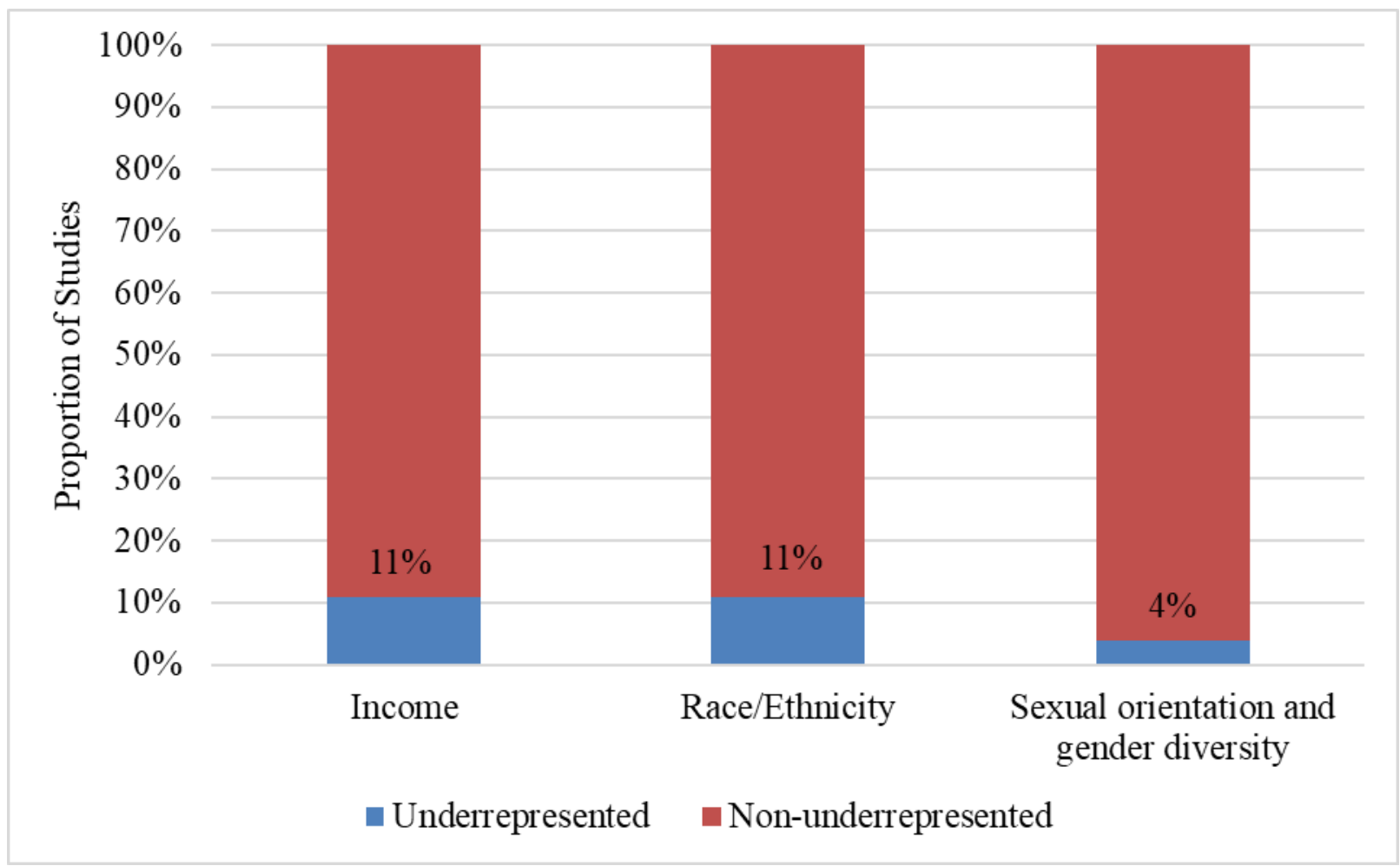

Note. $N=562$ U.S. based studies for Income, $N=562$ U.S. based studies for Race/Ethnicity, $N=771$ studies for Sexual orientation and gender diversity 


\section{Figure 5}

Proportion of Studies that Focused Primarily on an Underrepresented Group by Journal

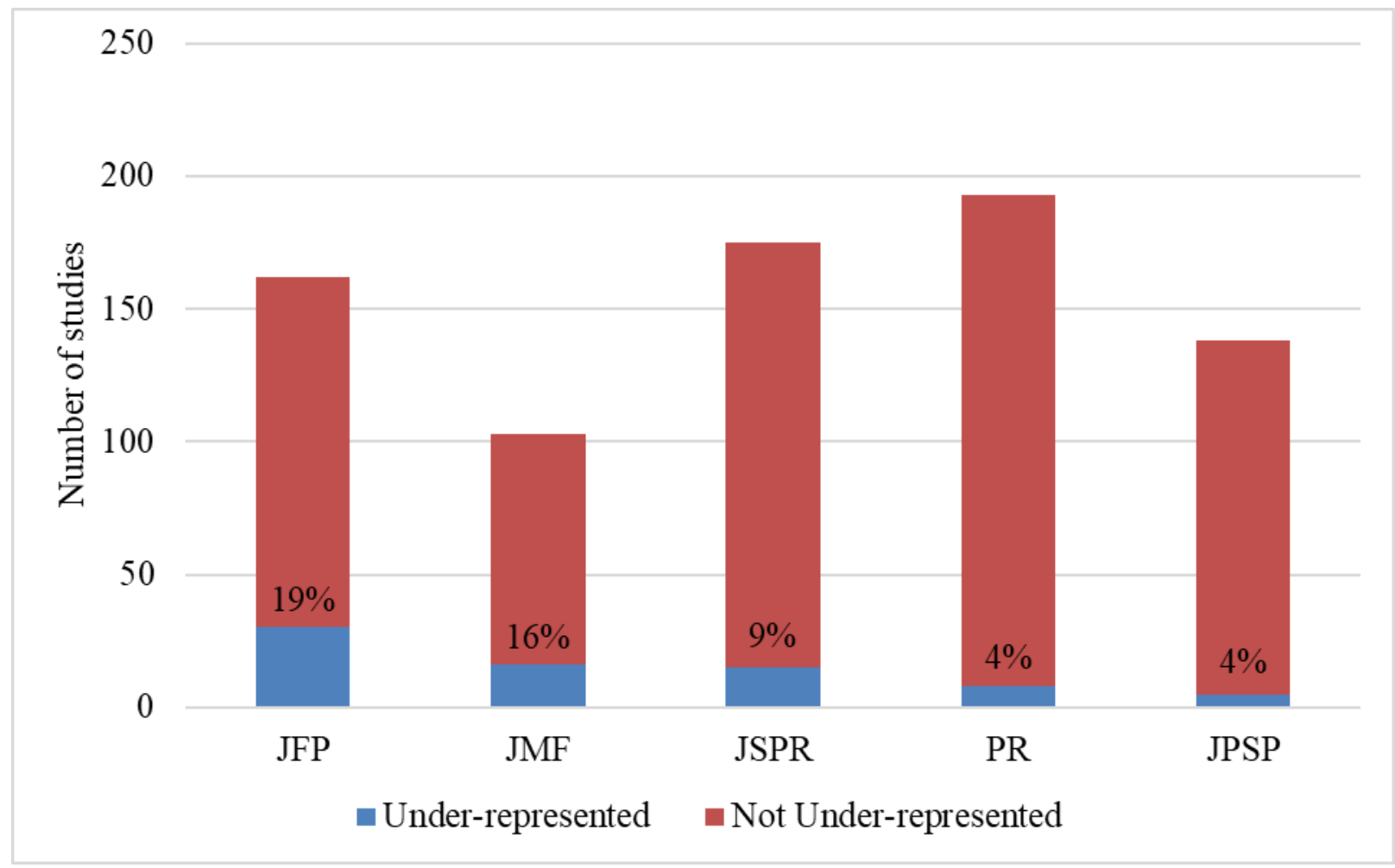

Note. Underrepresented includes the Primarily Low-income studies, Primarily Non-White studies, and Oversample or Exclusive SGM studies.

$\mathrm{JFP}=$ Journal of Family Psychology, JMF = Journal of Marriage and Family, JSPR = Journal of Social and Personal Relationships, PR = Personal Relationships, JPSP = Journal of Personality and Social Psychology: Interpersonal Relations and Group Processes section 